\title{
Effect of the CT Table Strap on Radiation Exposure and Image Quality during Cervical Spine CT
}

\author{
P.G. Kranz, J.D. Wylie, J.K. Hoang, and A.S. Kosinski
}

\begin{abstract}
BACKGROUND AND PURPOSE: The CT table strap may impair shoulder lowering during cervical spine CT. The purpose of this investigation was to evaluate the effect of the $\mathrm{CT}$ table strap on radiation exposure and image quality during $\mathrm{CT}$ of the cervical spine.
\end{abstract}

MATERIALS AND METHODS: Patients undergoing cervical spine CT were prospectively randomized to having the CT table strap placed around the torso and arms (control group) or around the torso only (intervention group). Radiation exposure, shoulder position, and image quality were evaluated. Potential confounders, including neck diameter and scan length, were also assessed.

RESULTS: Fifty-eight patients were enrolled and randomized, and 51 subjects were included in the final study population. There was a $21 \%$ decrease in radiation exposure in the intervention group compared with the control group (mean dose-length product, $540 \pm 152$ versus $686 \pm 200 \mathrm{mGy} \times \mathrm{cm}, P=.005)$. Subjects in the intervention group achieved shoulder lowering of an average of $>1$ vertebral body lower than the control group (mean shoulder level, $7.7 \pm 1.3$ versus $6.5 \pm 1.3, P=.001$ ). Subjective image quality, determined by the lowest level of spinal cord visibility, was also better in the intervention group (mean cord visibility level, $6.9 \pm 1.3$ versus $5.9 \pm 1.3, P=.006$ ). No differences in neck diameter $(P=.28)$ or scan length $(P=.55)$ were observed between groups.

CONCLUSIONS: The CT table strap inhibits shoulder lowering during CT of the cervical spine. Placement of the patient's arms outside the $\mathrm{CT}$ table strap results in decreased radiation exposure and increased image quality compared with patients whose arms are placed inside the strap.

ABBREVIATIONS: $\mathrm{DLP}=$ dose-length product; $\mathrm{CTDI}_{\mathrm{vol}}=$ volume $\mathrm{CT}$ dose index; $\mathrm{SSDE}=$ size-specific dose estimate

pr recent years, increasing radiation exposure from CT has prompted attention to dose-reduction strategies, both in the medical literature and by various professional societies. ${ }^{1,2}$ Several techniques to reduce the dose have been developed by the CT manufacturers, including tube current modulation, iterative reconstruction, and organ-based dose modulation. Many dose-reduction strategies involve trade-offs, however, including factors related to image quality, such as increased image noise or subjective alterations in

Received June 20, 2014; accepted after revision July 6.

From the Departments of Radiology (P.G.K., J.D.W., J.K.H.), Radiation Oncology (J.K.H.), and Biostatistics and Bioinformatics (A.S.K.), Duke University Medical Center, Durham, North Carolina.

Paper previously presented in part as an oral abstract at: Annual Meeting of the American Society of Neuroradiology and the Foundation of the ASNR Symposium, April 21-26, 2012; New York, New York.

Please address correspondence to Peter G. Kranz, MD, Department of Radiology, Duke University Medical Center, Box 3808, Durham, NC 27710; e-mail: peter.kranz@duke.edu

-- Indicates open access to non-subscribers at www.ajnr.org

Evidence-Based Medicine Level 1.

http://dx.doi.org/10.3174/ajnr.A4074 image appearance, and financial impacts related to product cost and supporting hardware requirements. ${ }^{3-5}$ Dose-reduction strategies that minimize these trade-offs are, therefore, clearly desirable.

One method for reducing radiation exposure without compromising image quality or increasing cost is through optimization of patient positioning. We have observed in practice, for example, that image quality in the lower cervical spine is dependent on the degree to which patients are able to lower their shoulders (Fig 1), because removal of the patient's shoulders from the imaged region decreases attenuation of the radiation beam. When automated tube current modulation is used, shoulder lowering also results in a reduction in the required tube current and, therefore, decreased radiation exposure. Our experience further suggests that 1 factor that affects shoulder lowering is the placement of the CT table strap. When the arms are placed within the strap, they may be constrained medially against the torso, causing the shoulders to elevate, thereby reversing previously performed shoulder-lowering maneuvers, and may impair further effort to shift the shoulders downward out of the imaging FOV.

The purpose of this study was to evaluate the effect of the table 

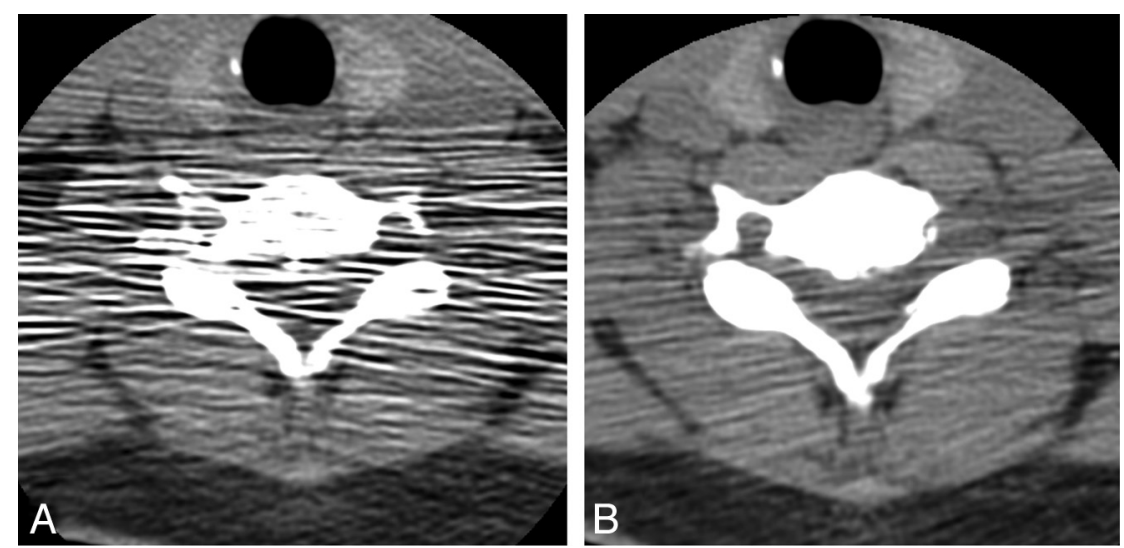

FIG 1. Effect of shoulder position on image quality in the lower cervical spine. Axial CT scan obtained in a 55-year-old man before $(A)$ and following $(B)$ shoulder lowering, demonstrating substantial improvement in image quality. The images were acquired only a few minutes apart, as part of an interventional procedure, by using otherwise identical scan techniques (CT fluoroscopy; $120 \mathrm{kVp} ; 60 \mathrm{~mA}$; 2.5-mm section thickness; 1-second rotation time; display FOV, $12 \mathrm{~cm}$ ).
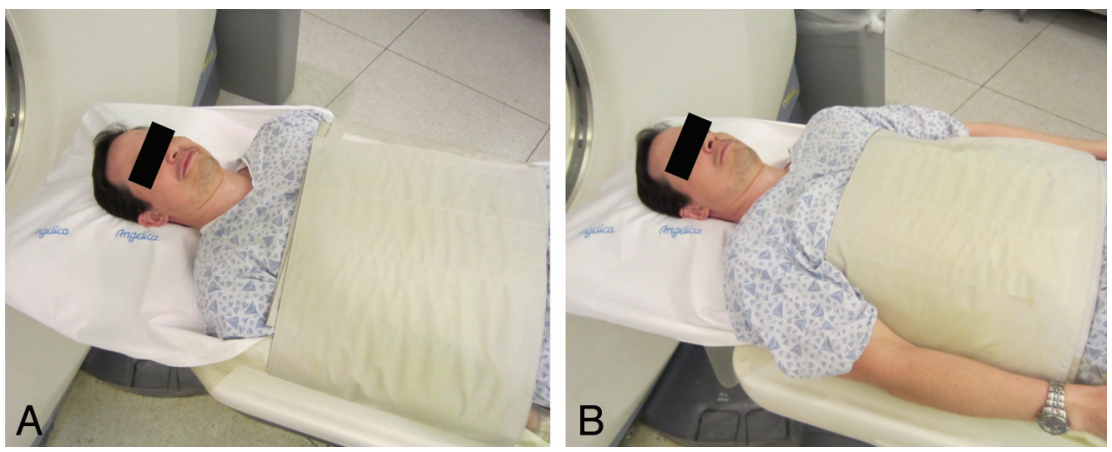

FIG 2. Position of the CT table strap relative to the arms in the control $(A)$ and intervention $(B)$ groups. Images were obtained by using an asymptomatic volunteer.

strap on radiation exposure during $\mathrm{CT}$ of the cervical spine. We hypothesized that by placing the arms outside the strap, patients would be able to increase shoulder lowering compared with patients whose arms remained inside the strap, resulting in decreased radiation exposure and improved image quality.

\section{MATERIALS AND METHODS}

This investigation was a prospective, randomized trial comparing cervical spine CTs obtained in patients referred from the emergency department by using 2 different positions of the CT table strap. The control group had the strap placed in the standard fashion, with the arms inside the strap, and the intervention group had the strap placed around the torso only, with the arms outside the strap (Fig 2). The study was approved by the local institutional review board and was compliant with Health Insurance Portability and Accountability Act regulations.

\section{Subjects}

Patients referred from the emergency department for cervical spine imaging with a study indication of suspected or observed trauma were eligible to participate if they met the following inclusion criteria: a conscious patient 1) able to follow commands and cooperate with instructions, 2) able to provide informed consent, 3 ) in a hard cervical collar, 4) male or nonpregnant females, and 5) 18 years of age or older. Patients who were unconscious, had sustained significant upper extremity injury (including, but not limited to, fractures of the shoulder or arm), had sustained significant injury to other internal organs or were otherwise unable to cooperate with instructions regarding shoulder lowering, were not in cervical collars, or were undergoing contrast examinations before the cervical spine CT were excluded. Patients were recruited during normal daytime hours on the basis of the availability of study personnel and were, therefore, not necessarily consecutive.

\section{Imaging Technique}

Eligible patients who consented to participation were randomized by using a predetermined simple randomization schedule. Subjects were all given the same instructions by the CT technologist and the study staff regardless of assigned study group. Subjects were first positioned on the CT table with shoulders lowered as much as possible per the standard imaging protocol. The CT technologist then placed the table strap according to the subject's randomized assignment. The strap was secured according to the technologist's standard practice, and no special instructions were provided regarding how securely it should be applied. The subject was told that he or she would receive further instructions regarding shoulder lowering and that he or she should maintain that position throughout the scan. Immediately before obtaining the scout images, the subject was verbally instructed to again lower his or her shoulders as much as possible. No immediate attempt was made to judge the degree of compliance with the instructions, and the instructions were not repeated or otherwise emphasized unless the subject indicated that he or she did not hear or understand the instructions.

All subjects were scanned by using 1 of two 64-section multidetector row CT scanners in the emergency department (LightSpeed VCT; GE Healthcare, Milwaukee, Wisconsin). After the scout image was obtained, the technologist prescribed the z-axis coverage according to the standard protocol. Before scanning, a study radiologist reviewed the $\mathrm{z}$-axis coverage to ensure coverage from the clivus through the T1 vertebral body, reducing or increasing the $\mathrm{z}$-axis coverage as necessary, to ensure that only the area of interest was scanned and that the scan coverage was as homogeneous as possible throughout the study population as a whole. The same scan protocol was used for all patients, by using the following parameters: $120 \mathrm{kV}$ (peak); automatic tube current modulation (tube current minimum, $150 \mathrm{~mA}$; maximum, 440 $\mathrm{mA}$ ); noise index, 10; axial scan mode; rotation time, 0.5 seconds; display FOV, $12 \mathrm{~cm}$; large-body scan FOV. Images were acquired at $0.625 \mathrm{~mm}$ and were reconstructed in the sagittal and coronal planes at 2-mm thickness by using a bone algorithm. 


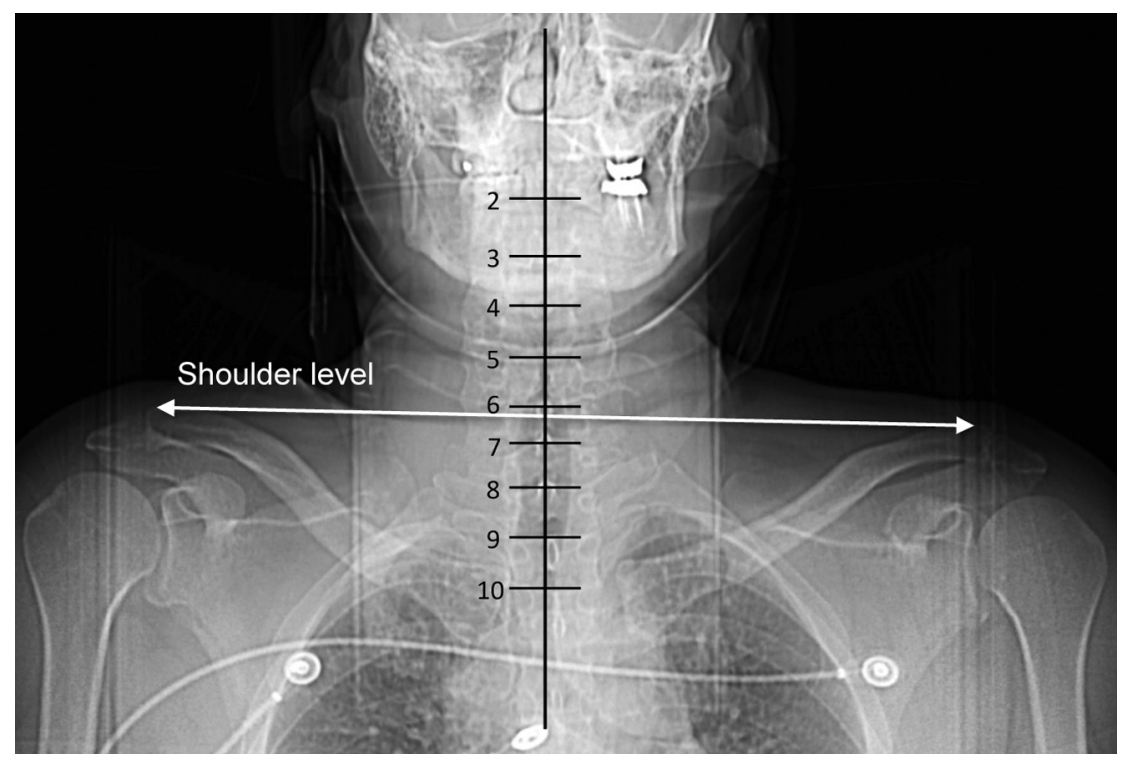

FIG 3. Determination of shoulder level. The level was defined on the anteroposterior scout image as the intersection of a line connecting the acromion processes with the spinal axis.

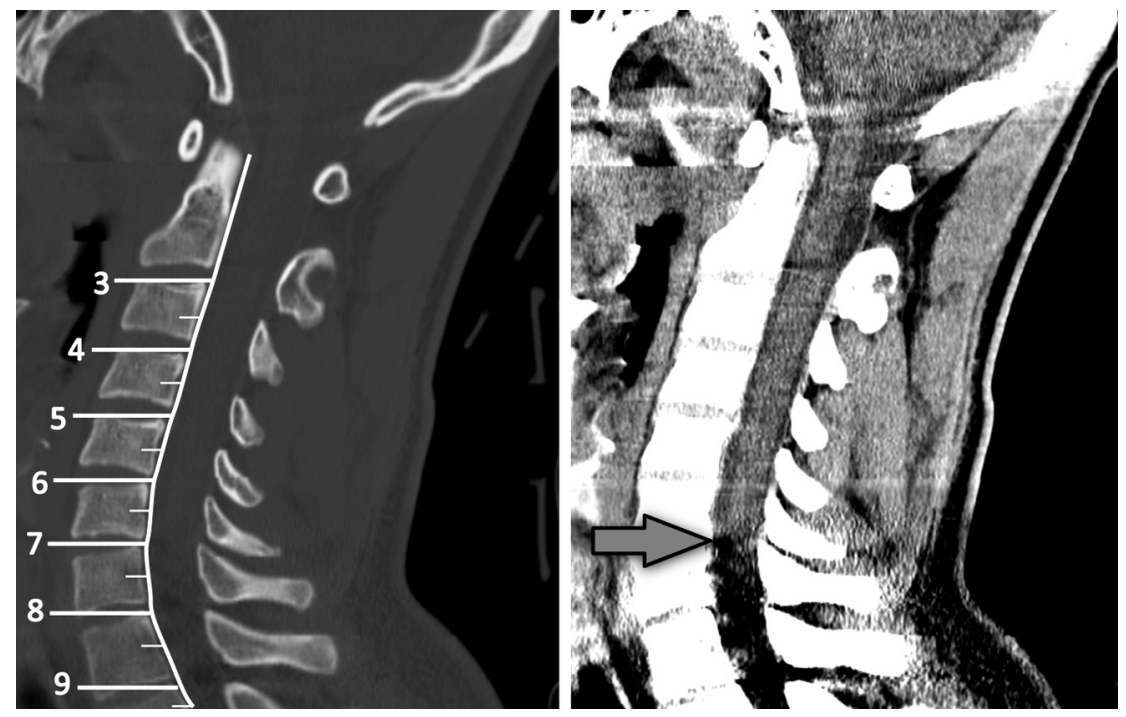

FIG 4. Method for grading spinal cord visibility. The level was defined as the lowest spinal level where the margins of the spinal cord were distinguishable from the adjacent CSF (arrow).

\section{Outcome Measures}

The primary outcome measure for the investigation was radiation exposure, measured by dose-length product (DLP). Secondary outcome measures included the anatomic level of the shoulders and subjective image quality, determined by visibility of the spinal cord.

\section{Data Analysis}

Image analysis was performed by a board-certified radiologist who held a Certificate of Added Qualification in neuroradiology and by a board-certified radiologist who was a fellow in neuroradiology. At the time of image analysis, the radiologists were blinded to the study group assignment. To ensure homogeneous scan lengths, subsequent to enrollment, subjects were excluded if the anatomic coverage of the scan extended below the T2-3 disk space or if a substantial portion of the T1 vertebral body ( $>25 \%$ total volume) was excluded from the scan.

DLP and volume CT dose index $\left(\mathrm{CTDI}_{\mathrm{vol}}\right)$ were recorded from the automated dose report generated by the scanner. A size-specific dose estimate (SSDE) was calculated for each subject according to the methods outlined in the American Association of Physicists in Medicine Report 204 by using the following equation:

$$
S S D E=f_{\text {size }}^{32, \text { Lat }} \times C T D I_{\text {vol }}^{32}
$$

where $f_{\text {size }}^{32 \text { Lat }}$ is the size-specific conversion factor for the lateral diameter measured on the scout image for a $32-\mathrm{cm}$ polymethylmethacrylate phantom and $C T D I_{\text {vol }}^{32}$ is the volume CTDI recorded from the patient's automated dose report. $^{6}$

Shoulder level was determined by creating a straight line on the anteroposterior scout image connecting the acromion processes bilaterally and evaluating the level where this line intersected the spine (Fig 3). A numeric value was assigned, depending on where the intersection occurred, with the superior endplate of the vertebral body assigned an integer value and fractional values assigned for lower portions of the vertebral body (eg, the superior endplate of $\mathrm{C} 7$ would be assigned 7.0; the midportion of $\mathrm{C} 7,7.5$; the superior endplate of T1, 8.0; and so forth).

Subjective image quality was measured on the sagittal reconstructions of the CT scan with a standard soft-tissue window/level setting (window: 342/ level: 56) as the lowest spinal level where the spinal cord could be discriminated from the CSF, by using the same numeric scoring system as that used for shoulder position (Fig 4).

To control for body habitus, we measured a potential confounder, neck size, by using the minimum transverse diameter of the neck on the anteroposterior scout image (Fig 5). Because scan length contributes to DLP, the total scan length was assessed for each subject by multiplying the total number of axial images acquired by the section thickness.

Image noise was measured by creating a standard-sized region of interest $\left(20 \pm 1 \mathrm{~mm}^{2}\right)$ in the tracheal air column on the $0.625-\mathrm{mm}$ axial CT source images at 3 different vertebral levels: 1 above the expected location of the shoulders (C4) and 2 closer to the expected shoulder level (C7 and T1). The noise was measured as the SD of the Hounsfield units within the 


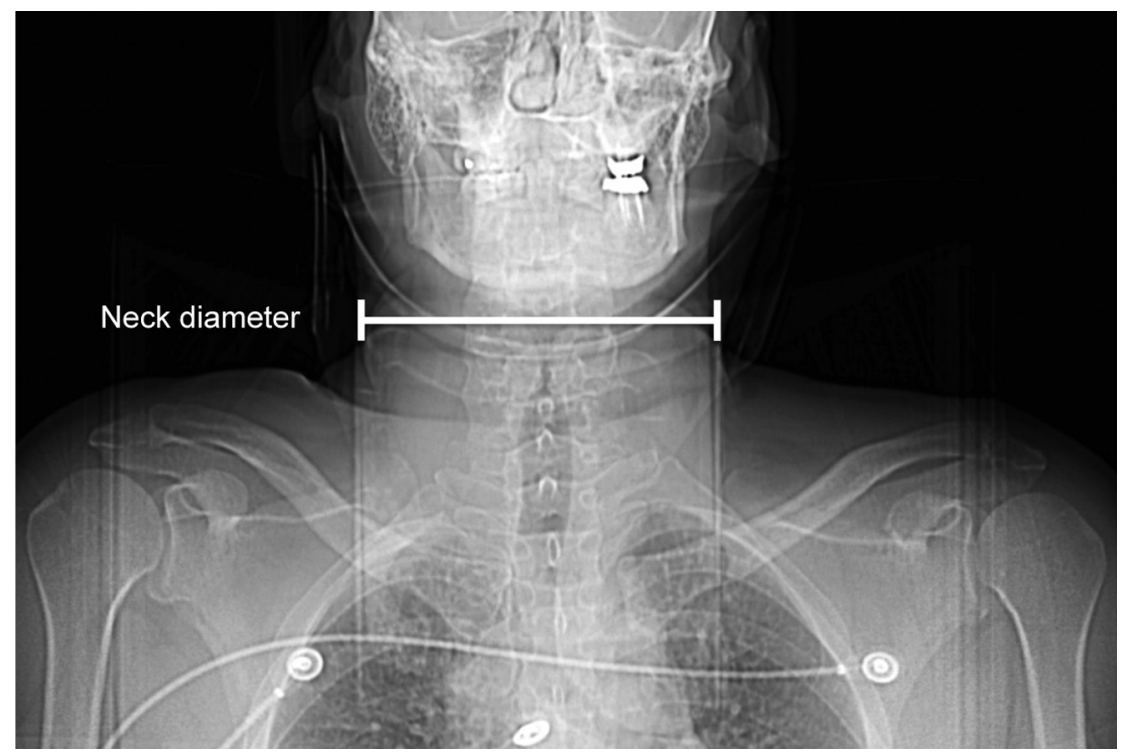

FIG 5. Determination of neck diameter based on the shortest transverse diameter measured on the anteroposterior scout image.

Table 1: Patient characteristics and scan length

\begin{tabular}{lccc}
\hline & $\begin{array}{c}\text { Control Group } \\
(\boldsymbol{n}=\mathbf{2 5})\end{array}$ & $\begin{array}{c}\text { Intervention } \\
\text { Group }(\boldsymbol{n}=\mathbf{2 6})\end{array}$ & $\begin{array}{c}\boldsymbol{P} \\
\text { Value }\end{array}$ \\
\hline Age (yr) & $49.2 \pm 21.8$ & $49.9 \pm 20.4$ & \\
Mean & 47 & 46 & $.87^{\mathrm{a}}$ \\
Median & $18-90$ & $19-88$ & \\
$\quad$ Range & $13(52.0)$ & $13(50.0)$ & \\
Sex & $12(48.0)$ & $13(50.0)$ & $1.0^{\mathrm{b}}$ \\
$\quad$ Male (No.) (\%) & & & \\
Female (No.) (\%) & $19.3 \pm 1.1$ & $19.1 \pm 1.4$ & \\
Scan length (cm) & 20 & 20 & $.55^{\mathrm{a}}$ \\
$\quad$ Mean & $16-20$ & $16-22$ & \\
Median & $13.2 \pm 1.4$ & $12.7 \pm 1.3$ & \\
Range & 13.0 & 12.5 & $.28^{\mathrm{a}}$ \\
Neck width (cm) & $11.1-17.8$ & $10.2-14.7$ & \\
$\quad$ Mean & & & \\
Median & & & \\
Range & & & \\
\hline
\end{tabular}

a Based on the 2-sample Wilcoxon test.

${ }^{\mathrm{b}}$ Based on the Fisher exact test.

region of interest. ${ }^{7}$ At each level, the measurement was obtained 3 times by using the same region of interest and an average noise was calculated. Demographic information (age and sex) was also collected.

\section{Statistical Analysis}

An enrollment target of 58 patients was selected so that the study would have a power of $80 \%$ to detect a difference in DLP values of $120 \mathrm{mGy} \times \mathrm{cm}$ (a 20\% difference, assuming a mean DLP of $600 \mathrm{mGy} \times \mathrm{cm}$ and $\sigma=175$ for both groups), with a 2-sided $\alpha=.05$.

The Shapiro-Wilk test was performed to determine the normality of the data and supported a normal distribution for the primary and secondary outcome measures, as well as $\mathrm{CTDI}_{\mathrm{vol}}$ and SSDE $(P$ values $=>.05)$; a 2 -tailed Student $t$ test was therefore used to compare these data. Patient age, neck width, scan length, and image noise were not normally dis- tributed (Shapiro-Wilk test, $P<.05$ ) and were evaluated by using a 2 -sample Wilcoxon test. The Fisher exact test was used to compare sex. Analysis was performed by using commercially available software (R, Version 2.8.1; http:// www.r-project.org/). A $P$ value of .05 was considered statistically significant.

\section{RESULTS}

Fifty-eight subjects were enrolled. After evaluation of the images, 3 patients from the control group and 4 from the intervention group were excluded due to excessive or inadequate anatomic coverage, resulting in a final cohort of 51 subjects, including 26 men and 25 women. Demographic characteristics and scan lengths are presented in Table 1. There were no differences in sex $(P=$ $1.0)$ or age ( $P=.87)$ between the control and intervention groups. The mean neck diameter was $13.2 \pm 1.4 \mathrm{~cm}$ for the control group and $12.7 \pm 1.3 \mathrm{~cm}$ for the intervention group, which was not significantly different $(P=$ .28). There was no difference in scan length between the 2 groups, with a mean scan length of $19.3 \pm 1.1 \mathrm{~cm}$ for the control group and $19.1 \pm 1.4 \mathrm{~cm}$ for the intervention group $(P=.55)$.

Comparison of radiation exposure and the estimated dose between the control and intervention groups is illustrated in Fig 6. Statistically significant decreases were observed in all metrics of radiation exposure in the intervention group compared with the control group: DLP decreased by $21 \%$ (mean DLP, $540 \pm 152$ versus $686 \pm 200, P=.005$ ), $\mathrm{CTDI}_{\mathrm{vol}}$ decreased by $28 \%$ (mean $\mathrm{CTDI}_{\mathrm{vol}}, 25.0 \pm 8.8$ versus $34.7 \pm 10.4 \mathrm{mGy}, P=.026$ ), and SSDE decreased by $17 \%$ (mean SSDE, $69.6 \pm 21.0$ versus $83.5 \pm 24.1$ mGy, $P=.032$ ).

Subjects in the intervention group were able to lower their shoulders an average of $>1$ vertebral body segment lower than subjects in the control group, with a mean shoulder level of $6.5 \pm$ 1.3 in the control group and $7.7 \pm 1.3$ in the intervention group $(P=.001)$. Subjective image quality was better in the lower portions of the spine as well, with visualization of the spinal cord an average of 1 vertebral segment lower in the intervention group compared with the control group, evidenced by a mean cord visibility level of $5.9 \pm 1.3$ in the control group versus $6.9 \pm 1.3$ in the intervention group $(P=.006)$. These results are illustrated in Fig 7.

Image noise at the various spinal levels is shown in Table 2. Noise was not statistically different between the 2 groups at any of the measured levels, though there was a trend toward decreasing $P$ values in the more inferior levels of the spine ( $P$ values at $\mathrm{C} 4, \mathrm{C} 7$, and $\mathrm{T} 1=.60, .49$, and .11 , respectively). Mean image noise was higher at the inferior vertebral levels, with a mean noise of $26.1 \pm$ 6.7, $28.9 \pm 9.6$, and $36.8 \pm 12.7$ at the C4, C7, and T1 levels, respectively. 

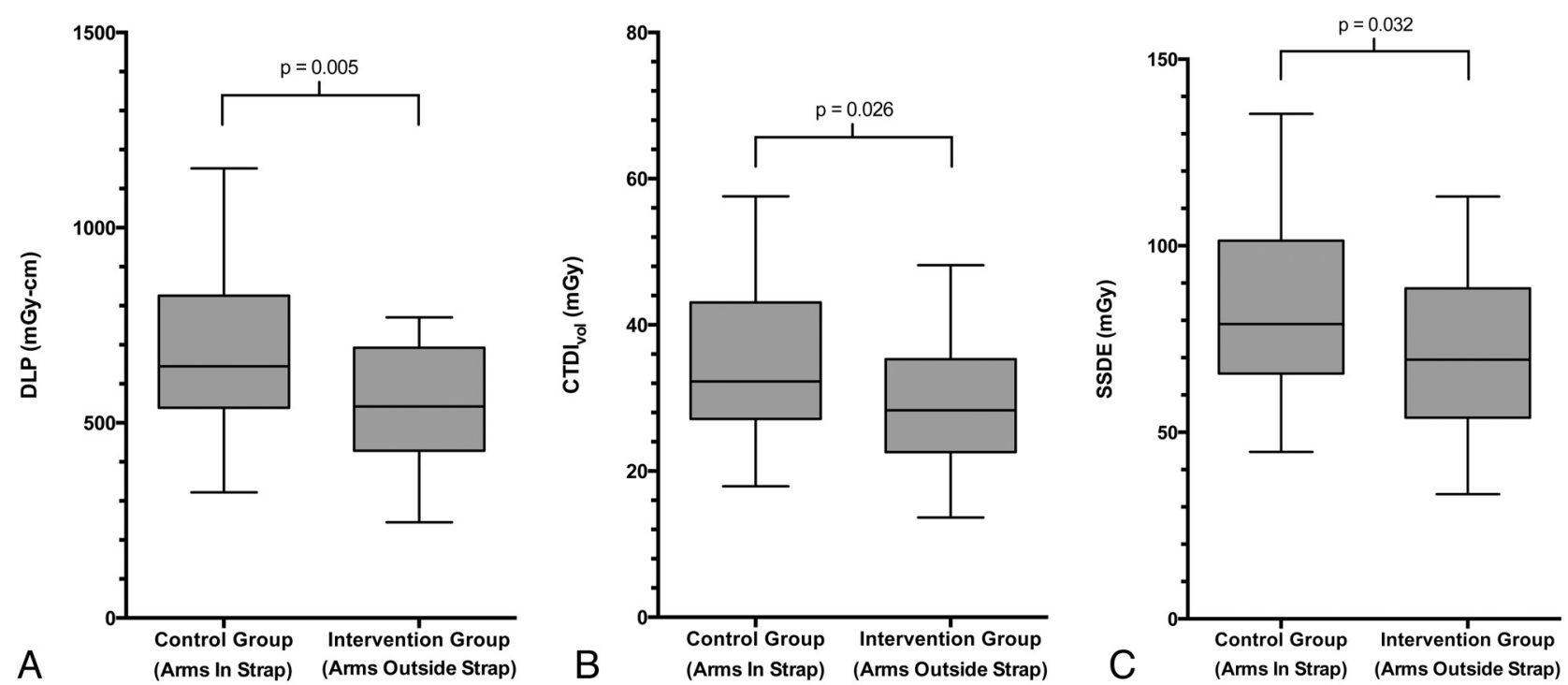

FIG 6. Box-and-whisker plots showing differences in $\operatorname{DLP}(A), \mathrm{CTDI}_{\mathrm{vol}}(B)$, and SSDE $(C)$ values in the control and intervention groups.
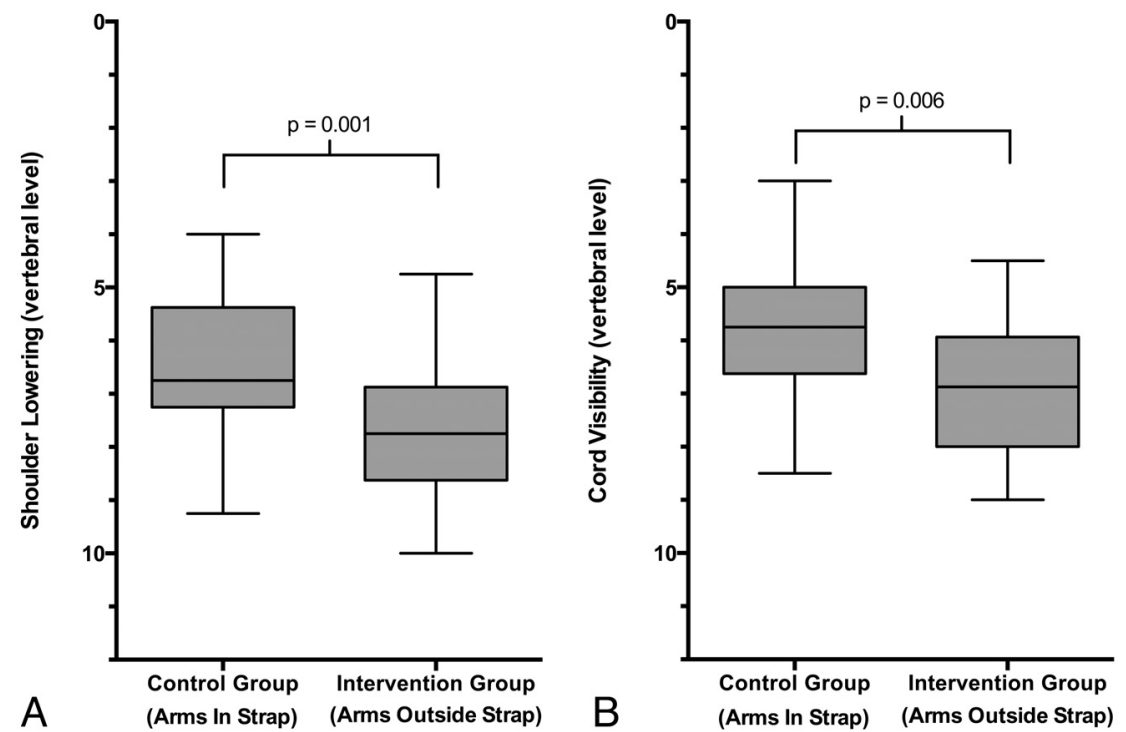

the product of $\mathrm{CTDI}_{\mathrm{vol}}$ and scan length. To ensure homogeneous scan lengths between groups, two potential sources of variability required attention in our investigation. First, there may be anatomic variability, with taller patients requiring a longer scan length to cover the same anatomy compared with shorter patients. Second, scan length is manually designated by the scanner operator and may therefore demonstrate scan-to-scan variation, depending on the operator.

We attempted to control for these potential confounders by using several methods, both before and following data acquisition. First, the study design was prospective, permitting randomization to reduce the effect of variations in individual subject anatomy. Second, at the scanner, z-axis coverage was manually determined for each subject by 1 of 2 study radiologists, according to predefined an-

FIG 7. Box-and-whisker plots showing differences in shoulder lowering $(A)$ and spinal cord visibility $(B)$ in the control and intervention groups. Note that the vertical axis has been reversed to correspond with the anatomic orientation of the vertebral levels.

\section{DISCUSSION}

Our investigation found that in patients undergoing cervical spine CT, shoulder lowering is impaired by placing the patient's arms inside the CT table strap and that improved shoulder lowering can easily be achieved by placing the strap around only the torso, allowing the arms to remain free. This simple modification resulted in a decrease in radiation exposure of $>20 \%$ in our study. Additionally, we found a subjective improvement in image quality in the lower cervical spine as well. This technique can be easily implemented into routine practice when scanning cooperative patients without the addition of any financial cost or logistic complexity.

DLP was used in this investigation as the primary outcome measure. DLP is a reflection of the total amount of radiation used in a CT examination that takes into account scan length, but it does not reflect the individual patient dose. ${ }^{8}$ It is derived by taking atomic criteria to reduce interoperator variability. Finally, subsequent to data acquisition, scans were reviewed to make sure underscanning or overscanning had not occurred, and subjects whose scans showed anatomic coverage outside predetermined parameters were excluded. These measures were ultimately effective in ensuring equal scan lengths between the 2 study groups.

$\mathrm{CTDI}_{\mathrm{vol}}$, a component of DLP, is a reflection of scanner radiation output. ${ }^{9}$ CTDI $_{\mathrm{vol}}$ estimates are based on uniform phantoms; thus, these do not take variations in individual body habitus into account. As a result, $\mathrm{CTDI}_{\mathrm{vol}}$ can underestimate dose by $40 \%-70 \%$, depending on age and body size. ${ }^{10}$ Size-specific doses have been proposed that rely on corrections to $\mathrm{CTDI}_{\mathrm{vol}}$ based on direct anatomic measurements of individual patients being scanned to minimize inaccuracies related to habitus. ${ }^{6}$ These SSDEs have been shown to more accurately represent the 
Table 2: Comparison of image noise by vertebral level

\begin{tabular}{cccc}
\hline $\begin{array}{l}\text { Image } \\
\text { Noise }\end{array}$ & $\begin{array}{c}\text { Control } \\
\text { Group }\end{array}$ & $\begin{array}{c}\text { Intervention } \\
\text { Group }\end{array}$ & $\begin{array}{c}\boldsymbol{P} \\
\text { Value }^{\mathbf{a}}\end{array}$ \\
\hline At C4 & & & \\
Mean & $26.8 \pm 6.2$ & $25.4 \pm 7.1$ & \\
Median & 27.9 & 26.5 & .60 \\
Range & $14.4-37.8$ & $5.7-34.3$ & \\
At C7 & & $27.7 \pm 8.3$ & \\
Mean & $30.2 \pm 10.7$ & 26.4 & .49 \\
Median & 29.9 & $15.9-48.9$ & \\
Range & $13.8-58.3$ & & \\
At T1 & & $34.7 \pm 14.9$ & \\
Mean & $39.0 \pm 9.8$ & 32.6 & .11 \\
Median & 37.6 & $7.3-70.1$ & \\
Range & $24.1-66.4$ & & \\
\hline
\end{tabular}

a Based on the 2-sample Wilcoxon test.

absorbed dose compared with $\mathrm{CTDI}_{\mathrm{vol}}{ }^{10}{ }^{10}$ In our investigation, both $\mathrm{CTDI}_{\mathrm{vol}}$ and SSDE were lower in the intervention group, with a $28 \%$ reduction in $\mathrm{CTDI}_{\mathrm{vol}}$ and a $17 \%$ reduction in SSDE observed. Furthermore, we found no difference in body habitus, as determined by transverse neck diameter, between our study groups. Together with the DLP reductions, these data demonstrate that the observed decreases in radiation exposure and dose metrics in the intervention group are attributable to the study intervention (eg, placement of the CT table strap) rather than to other factors such as variation in scan length or habitus.

In addition to differences in radiation, we also found differences in image quality between groups, with improved visibility of the lower spinal cord in the intervention group. This finding was despite the fact that automated tube current modulation should function to maintain image quality when attenuation of the $\mathrm{x}$-ray beam by soft tissue increases, such as in elevated shoulders. To quantitatively examine this finding, we measured image noise at several spinal levels both above and at the expected level of the shoulders, to determine the degree to which automated tube current modulation was effectively compensating for increased soft tissue around the cervicothoracic junction. We observed a nonsignificant trend toward increasing noise at the $\mathrm{C} 7$ and $\mathrm{T} 1$ levels in the control group. This observation is likely explained by the fact that the control group had a higher average shoulder level than the intervention group, resulting in a greater likelihood of reaching the maximum tube current during scanning at the lower cervical and upper thoracic levels, beyond which automated tube current modulation would no longer keep image noise constant.

Although there are no prior studies that evaluate the effect of shoulder positioning during cervical spine CT, it is well-known that arm position should be up for CT of the chest and down for CT of the neck or cervical spine, to reduce streak artifacts and dose when automated tube current modulation is used. A previous retrospective study of patients undergoing thoracoabdominal CT imaging in the setting of trauma investigated the effect of raising the arms outside the imaged volume. ${ }^{11}$ This investigation found that while image quality was tolerable with the arms down, raising the arms had the effect of improving image quality and reducing dose, with the trade-off of requiring longer scanner time because it necessitated a second-pass scan to image above the cervicothoracic junction. The magnitude of dose reduction in this study was $18 \%-45 \%$, depending on whether 1 or both arms were raised.
Another investigation advocated that a swimmers position be used during CT to improve visualization of the lower cervical spine. ${ }^{12}$ Because this technique required a second scan with the modified arm positioning as well, the authors recommended that the technique be used only as a problem-solving tool in select cases. Unlike these investigations, however, placement of the patient's arms outside the CT table strap does not increase scan time or require repeat scanning and can be routinely implemented for most patients undergoing cervical CT.

Our investigation has several limitations. First, the assessment of image quality in this investigation was based on visualization of the spinal cord, a feature that may not directly reflect the diagnostic performance of the scan in the detection of fractures, which is the primary purpose of obtaining cervical spine CT in the setting of trauma. However, the incidence of fractures, especially those of the lower cervical spine whose visualization may be impaired by the position of the shoulders, would be expected to be very low in light of the widespread use of cervical CT as the first-line imaging technique when screening patients with trauma, and a very large number of subjects would be needed to find enough fractures to sufficiently compare performance between groups. Nevertheless, the primary purpose of this investigation was to evaluate radiation exposure; therefore, the study size was selected to evaluate this end point. Second, we evaluated 1 scanner type with 1 model of table strap. Although different table strap configurations may be available from other vendors, including straps of varying widths or shapes, we would expect the effect on shoulder lowering to be similar in all straps that constrain the arms against the torso, regardless of strap shape. Third, this investigation was performed at 1 center, and though we do not have normative data for neck diameter for the population in general, our study group may include patients with a larger body habitus than at other centers. This could potentially overestimate the effect of dose reduction compared with a population of thinner patients because the ability to voluntarily lower one's shoulders when the arms are placed inside the restraint strap may be less inhibited in patients with a small body habitus.

Next, we used transverse neck diameter rather than body mass index to assess the homogeneity of body habitus between groups, to evaluate the effectiveness of our randomization, because body mass index was not available in all patients. However, it has been previously shown that transverse neck diameter correlates well with image quality and radiation dose during routine multidetector row CT scanning when automated tube current modulation is used and that region-specific measurements are better indicators of habitus than body mass index when optimizing cross-sectional imaging. ${ }^{13}$ Finally, SSDE measurements were developed for use in the torso, where anatomic geometry is less complex than at the cervicothoracic junction, ${ }^{6}$ and transverse neck diameter was used to calculate the SSDE, which may result in increased variability compared with a combination of transverse and anteroposterior measurements. ${ }^{10}$

\section{CONCLUSIONS}

In summary, our investigation has demonstrated that the CT table strap inhibits shoulder lowering during CT of the cervical spine. Placement of the patient's arms outside the strap facilitates shoul- 
der lowering, resulting in decreased radiation exposure and increased image quality. This simple change in positioning provides a mechanism for dose reduction that can be easily implemented when scanning cooperative patients and results in increased image quality without requiring data postprocessing or increasing cost.

\section{REFERENCES}

1. Costello JE, Cecava ND, Tucker JE, et al. CT radiation dose: current controversies and dose reduction strategies. AJR Am J Roentgenol 2013;201:1283-90

2. Goske MJ, Applegate KE, Boylan J, et al. The Image Gently campaign: working together to change practice. AJR Am J Roentgenol 2008;190:273-74

3. Hara AK, Paden RG, Silva AC, et al. Iterative reconstruction technique for reducing body radiation dose at $\mathrm{CT}$ : feasibility study. AJR Am J Roentgenol 2009;193:764-71

4. Silva AC, Lawder HJ, Hara A, et al. Innovations in CT dose reduction strategy: application of the adaptive statistical iterative reconstruction algorithm. AJR Am J Roentgenol 2010;194:191-99

5. Yu L, Bruesewitz MR, Thomas KB, et al. Optimal tube potential for radiation dose reduction in pediatric CT: principles, clinical implementations, and pitfalls. Radiographics 2011;31:835-48

6. Boone JM, Strauss KJ, Cody DD, et al. Size-Specific Dose Estimates
(SSDE) in Pediatric and Adult Body CT Examinations: Report of AAPM Task Group 204. College Park, Maryland: American Association of Physicists in Medicine; 2011

7. Schindera ST, Nelson RC, Toth TL, et al. Effect of patient size on radiation dose for abdominal MDCT with automatic tube current modulation: phantom study. AJR Am J Roentgenol 2008;190: W100-05

8. Huda W, Mettler FA. Volume CT dose index and dose-length product displayed during CT: what good are they? Radiology 2011; 258:236-42

9. McCollough $\mathrm{CH}$, Leng S, Yu L, et al. CT dose index and patient dose: they are not the same thing. Radiology 2011;259:311-16

10. Brady SL, Kaufman RA. Investigation of American Association of Physicists in Medicine Report 204 size-specific dose estimates for pediatric CT implementation. Radiology 2012;265:832-40

11. Brink M, de Lange F, Oostveen LJ, et al. Arm raising at exposurecontrolled multidetector trauma CT of thoracoabdominal region: higher image quality, lower radiation dose. Radiology 2008;249: 661-70

12. Kane AG, Reilly KC, Murphy TF. Swimmer's CT: improved imaging of the lower neck and thoracic inlet. AJNR Am J Neuroradiol 2004;25:859-62

13. Hoang JK, Gafton AR, Eastwood JD, et al. Correlation of cross-sectional diameter with image quality and radiation exposure in MDCT examinations of the neck. AJR Am J Roentgenol 2011;197: W904-09 